\title{
Influence of sex on diaphragm thickness using a method for determining intima media thickness in healthy young adults
}

Masayoshi Oguri ( $\sim$ ooguri-m@chs.pref.kagawa.jp )

Kagawa Prefectural University of Health Sciences

Tohru Okanishi

Tottori University

Takuya Ikeguchi

Tottori University Hospital

Kaoru Ogo

Kagawa Prefectural University of Health Sciences

Sotaro Kanai

Tottori University

Yoshihiro Maegaki

Tottori University

Shinichi Wada

Tenri Health Care University

Takashi Himoto

Kagawa Prefectural University of Health Sciences

\section{Research Article}

Keywords: diaphragm thickness, ultrasonography, respiratory muscle strength, spine position

Posted Date: October 28th, 2021

DOI: https://doi.org/10.21203/rs.3.rs-1014391/v1

License: (1) (1) This work is licensed under a Creative Commons Attribution 4.0 International License.

Read Full License 


\section{Abstract}

Background: To clarify the differences in diaphragm thickness between male and female participants in healthy young adults using ultrasonography and to investigate the relationship between diaphragm thickness and respiratory pressure.

Methods: Twenty-nine healthy individuals (16 females and 13 males) participated in the study. Diaphragm thickness was measured at total lung capacity (TLC) and at functional residual capacity (FRC) in each participant. We measured the diaphragm thickness using a method for mean intima media thickness. Moreover, change ratio of diaphragm thickness was calculated with the diaphragm thickness at TLC and FRC.

Results: Mean diaphragm thicknesses at FRC in males were significantly narrower than those in females $(p<0.001)$. The change ratio of diaphragm thickness was significantly augmented in males compared with that in females $(p<0.001)$. There was a significant positive correlation between the change ratio of diaphragm thickness and respiratory muscle strength in males but not in females.

Conclusions: The change ratio might be an alternative to evaluate respiratory muscle strength in young adult males. The diaphragm thickness values and the change ratio of diaphragm thickness in healthy young adults, evaluated by B-mode sonography, were varied based on sex.

\section{Background}

Diaphragm movement and function can be assessed through various methods, including chest X-ray, pulmonary function test, maximum respiratory pressure, and electromyography. Diaphragm ultrasonography is a cost-beneficial method that enables easy assessment of diaphragm thickness; moreover, it is a noninvasive bedside tool that can assess diaphragm function and movement [1]. At the bedside, we can measure diaphragm thickness using the B-mode with a linear probe, and diaphragm movement using M-mode with a convex probe, via ultrasonography [2, 3]. Recently, many reports have demonstrated associations among respiratory muscle strength, neuromuscular disorders, and chronic obstructive pulmonary disease assessed using ultrasonography [4, 5]. However, few studies have been published regarding the difference in diaphragm thickness between the sexes.

The respiratory pressure test is a useful method to assess respiratory muscle strength in clinical practice [6]. The evaluation of respiratory pressure is often used to determine muscle weakness in patients with muscular dystrophy, assess disease severity, and postulate prognosis [7]. Several studies were performed to define normative values for maximal respiratory pressure in children and adults, considering factors such as age, sex, height, and body weight [8, 9]; however, there are no reports regarding the relationship between diaphragm thickness using ultrasonography and maximal respiratory pressure in healthy young adults. 
This study aimed to determine diaphragm thickness using ultrasonography in male and female healthy young adults. Additionally, we investigated the relationship between diaphragm thickness and maximal respiratory pressure.

\section{Methods}

\section{Participants}

This study was a prospective observational study conducted from October 2016 to March 2019. The study was approved by the Institutional Review Board of the Kagawa Prefectural University of Health Sciences, and informed consent was obtained from the participants before the study. Additionally, the study was conducted in accordance with the Declaration of Helsinki. Twenty-nine healthy participants (16 females, 13 males) aged 19-34 years were enrolled. The participants were either students or staff at Kagawa Prefectural University of Health Sciences and Tottori University. Body weight and height were measured, and body surface and body mass index (BMI) were calculated for each participant. Participants with obesity $(\mathrm{BMI}>25)$, neuromuscular disease, cardiorespiratory disease, or chronic illness were excluded.

\section{Ultrasound imaging and analysis}

Using an ultrasound machine (ARIETTA 60, Hitachi, Chiba, Japan), measurements were taken with the 7.5 $\mathrm{MHz}$ linear array transducer placed in the $9^{\text {th }}$ or $10^{\text {th }}$ intercostal space between anterior and midaxillary lines in the zone of apposition. The participants were placed in the supine position for the measurement [10]. Only one focus-level measurement was chosen, and the depth was at diaphragm level. Mean diaphragm thickness (MDT) was measured using the mean intima media thickness (IMT) method both at total lung capacity (TLC) and at functional residual capacity (FRC) [11]. The mean IMT method is often used for measuring plaques automatically in the carotid artery. We calculated a mean of three points using IMT software: the center of the diaphragm thickness, and two surrounding points on each side (1 $\mathrm{cm}$ from the center of the diaphragm thickness) (Fig. 1).

Subsequently, we calculated the change ratio of diaphragm thickness (expressed in percentage) using the following formula: MDT at TLC minus MDT at FRC divided MDT at FRC [(MDTtlc - MDTfrc)/ MDTtlc] $\times 100$.

\section{Measurement of pulmonary function test and maximal respiratory pressure}

A multifunctional spirometer (Autospiro AS507, Minato Medical Science, Osaka, Japan) was used to evaluate respiratory function. Before recording respiratory pressure data, the spirometric parameters of forced vital capacity (FVC) and forced expiratory volume $\%$ in one second $\left(\mathrm{FEV}_{1}\right)$ were evaluated with 
participants in the sitting position. Respiratory pressure examinations, as well as the spirometric examination, were also conducted with the participants in the sitting position. First, to measure exhaled air from the participant's mouth, they had to hold a mouthpiece between their teeth. Second, they held the cylinder between their teeth using light pressure, and a clip was placed on their nose to prevent air leaking out during the respiratory tests. Finally, we recorded maximal respiratory pressure to assess respiratory muscles strength. We measured maximum expiratory pressure (MEP) or maximum inspiratory pressure (MIP) by asking participants to breath in/out as deeply as they could and breath out/in sharply and quickly. The MEP and MIP were recorded three times, and maximal end-expiratory and end-inspiratory potential data were chosen for further analysis.

\section{Statistical analysis}

We compared the age, weight, height, BMI, body surface, mean diaphragm thickness, change ratio of the mean diaphragm thickness, and maximal end-expiratory and end-inspiratory potentials between males and females. We used the Welch t-test for these analyses. Analyses were performed using GraphPad 6 (GraphPad Software, La Jolla, CA, USA). Pearson's correlation coefficient was used to assess correlation between diaphragm measurements and the maximal respiratory potentials or anthropometric measurements. A p-value $<0.05$ was considered statistically significant.

\section{Results}

Participant baseline characteristics by sex are shown in Table 1. Sixteen participants (57\%) were female, with a mean age of 24 (interquartile range [IQR]: 19-34) years, mean weight of 51 (IQR: 41-58) kg, and mean height of 158 (IQR: 151-165) cm. The mean BMI of the female participants was 20 (IQR: 18-24) $\mathrm{kg} / \mathrm{m}^{2}$. The height, weight, and BMI of males were higher than those of females. There were no significant differences with respect to sex in age, vital capacity, and $\mathrm{FEV}_{1}$.

The MDT at FRC, MDT at TLC, and change ratio of diaphragm thickness are listed in Table 2. The MDTs at FRC and TLC in the male participants were 1.2 (IQR: 0.6-1.5) and 3.8 (IQR: 2.5-5.5), respectively. The MDTs at FRC and TLC in female participants were 1.5 (IQR: 1.3-2.1) and 3.7 (IQR: 2.3-5.2), respectively. The mean change ratio of the diaphragm thickness was $67.2 \%$ (IQR: $58.2-77.6$ ) in males and $57.2 \%$ (IQR: 40.0-72.1) in females. Although the MDT at FRC in males was significantly narrower than in females $(1.2 \pm 0.3 \mathrm{~mm}$ vs $1.5 \pm 0.2, p<0.001)$, the change ratio of diaphragm thickness in males was larger than that in females $(67.2 \pm 6.2 \%$ vs $57.2 \pm 7.8 \%, p<0.001)$. There were no significant differences in the MDT at TLC with respect to sex.

Table 2. Ultrasound values of right diaphragm thickness 


\begin{tabular}{|llll|}
\hline Variable (Mean \pm SD) & Male & Female & p-value \\
\hline Diaphragm thickness at functional residual capacity $(\mathrm{mm})$ & $1.2 \pm 0.3$ & $1.5 \pm 0.2$ & $<0.001$ \\
\hline Diaphragm thickness at total lung capacity $(\mathrm{mm})$ & $3.8 \pm 0.8$ & $3.7 \pm 0.8$ & 00.82 \\
\hline Change ratio of diaphragm thickness $(\%)$ & $67.2 \pm 6.2$ & $57.2 \pm 7.8$ & $<0.001$ \\
\hline
\end{tabular}

Table 3 shows the mean maximal respiratory pressures in male and female participants. The mean MEPs were 88.2 (IQR: 49.7-113.6) $\mathrm{cmH}_{2} \mathrm{O}$ and 56.8 (IQR: 32.3-87.0) $\mathrm{cmH}_{2} \mathrm{O}$ and mean MIPs were 79.0 (IQR: 44.9-116.1) $\mathrm{cmH}_{2} \mathrm{O}$ and 81.1 (IQR: $\left.43.5-129.6\right) \mathrm{cmH}_{2} \mathrm{O}$ in male and female participants, respectively. While there were no significant differences in MIPs, the MEPs were higher in males than in females.

Table 3. Maximal respiratory pressures

\begin{tabular}{llll} 
Variable $($ Mean \pm SD $)$ & Male & Female & p-value \\
\hline Maximal expiratory pressure $\left(\mathrm{cmH}_{2} \mathbf{0}\right)$ & $88.2 \pm 22.5$ & $56.8 \pm 16.2$ & $<0.001$ \\
\hline Maximal inspiratory pressure $\left(\mathrm{cmH}_{2} \mathbf{0}\right)$ & $79.0 \pm 23.8$ & $81.1 \pm 26.0$ & 0.81 \\
\hline
\end{tabular}

SD, standard deviation

Subsequently, we assessed the correlation between the change ratio of diaphragm thickness and anthropometric data or pulmonary function test or maximal respiratory mouth pressure. Significant positive correlations were found only in males between the change ratio of diaphragm thickness and $\% \mathrm{FVC}(r=0.57, p=0.04)$, the change ratio of the diaphragm thickness and MIP $(r=0.56, p=0.05)$, and the change ratio of the diaphragm thickness and MEP $(r=0.71, p=0.007)$ (Fig. 2). By contrast, there were no significant correlations in females between the change ratio of diaphragm thickness and maximal respiratory mouth pressures, or between change ratio of diaphragm thickness anthropometric data.

\section{Discussion}

In this study, we determined normal reference values for diaphragm thickness in healthy young adults using ultrasound to assess respiratory muscle strength; additionally, we analyzed the relationship between diaphragm thickness and maximal respiratory mouth pressure by classifying the participants by sex. Our study revealed a significant positive correlation between the change ratio of diaphragm thickness and respiratory muscle strength in young adult males.

Previous studies have demonstrated various methods to observe diaphragm thickness with ultrasonography $[1,12]$. Regarding the studies on diaphragm thickness with ultrasonography, Boon et al. reported a lower limit of normal diaphragm thickness with ultrasonography placed over one of the most 
caudal intercostal spaces of $1.4 \mathrm{~mm}$ in participants between 33 and 84 years of age [3]. De Bruin et al., using ultrasonography to place the right hemidiaphragm at the apposition zone, reported a mean normal diaphragm thickness at TLC for participants aged 7-12 years of $3.5 \mathrm{~mm}$ [13]. Our study revealed that the lower limit of the diaphragm thickness at FRC in males and females was $1.2 \mathrm{~mm}$ and $1.5 \mathrm{~mm}$, respectively. Additionally, the mean normal diaphragm thickness at TLC in males was $3.8 \mathrm{~mm}$. These results were approximately consistent with previous studies $[3,13]$.

Measuring respiratory mouth pressure can contribute to the assessment of respiratory muscle strength in neuromuscular disease. The mean respiratory muscle strength values have been previously reported [7, $14,15]$. The mean MIP and MEP for participants aged $>18$ years were 106 and $148 \mathrm{cmH}_{2} \mathrm{O}$ in males and 73 and $93 \mathrm{cmH}_{2} \mathrm{O}$ in females, respectively [15]. Gibson et al. collected data from a small group of 10 healthy Caucasian women, obtaining an MEP of $67-140 \mathrm{cmH}_{2} \mathrm{O}$ and MIP of 35-95 $\mathrm{cmH}_{2} \mathrm{O}$, respectively [16]. Our results are similar to these results. Additionally, Heinzmann-Filho et al., despite the lack of statistical differences, found higher respiratory muscle strength values for boys than girls [6]. Our results also showed a higher MEP in males than in females. As for MIP, no significant difference was found between the sexes. To assess the MEP in young adults, we should evaluate the measurement data according to sex.

The change ratio of the diaphragm thickness appears to be a useful indicator to evaluate morphological changes of the diaphragm and differentiation based on sex. Previous studies on diaphragm thickness with ultrasonography based on sex in healthy infants and children indicated no significant differences between males and females [17]. In an adult study, Boussuges et al. found weak correlations between diaphragmatic excursion and body weight or height [18], and significant correlations between diaphragmatic measurements and $\mathrm{FEV}_{1}[5,19]$. Additionally, there was a significant relationship between MEP and body weight [6]. In this study, a significant positive correlation was observed between the change ratio of diaphragm thickness and respiratory muscle strength or FVC in young adult males. This result suggests that the change ratio of the diaphragm thickness correlates with breathing effort. The change ratio of diaphragm thickness might be an alternative to evaluate respiratory muscle strength in young adult males.

Our study has some limitations. First, the sample size was small, and we only assessed young adults. A larger sample size with various age groups is necessary to draw more definitive conclusions. Second, a diaphragm measurement methodology using the IMT method has never been reported; therefore, we could not compare the diaphragm thickness results using the IMT method with previous data. Further studies are required to determine the optimal diaphragm assessment method with ultrasonography.

\section{Conclusions}

In conclusion, diaphragm sonography could enable easy assessment of diaphragm thickness. Normal reference values based on sex for diaphragm thickness and change ratio of the diaphragm thickness, evaluated by B-mode sonography in healthy young adults, were determined in this study. A significant 
positive correlation was found between the change ratio of diaphragm thickness and respiratory muscle strength in young adult males; thus, the change ratio of diaphragm thickness may be an alternative indicator to evaluate respiratory muscle strength.

\section{Abbreviations}

BMI, body mass index; FEV, forced expiratory volume; FRC, functional residual capacity; FVC, forced vital capacity; IMT, intima media thickness; IQR, interquartile range; MDT, mean diaphragm thickness; MEP, maximum expiratory pressure; MIP, maximum inspiratory pressure; TLC, total lung capacity

\section{Declarations}

Not applicable.

\section{Ethics approval and consent to participate}

This study was approved by the Institutional Review Board of the Kagawa Prefectural University of Health Sciences. Informed consent was obtained from all patients for being included in the study. Additionally, written informed consent was obtained from all subjects.

\section{Consent for publication}

Not applicable.

\section{Availability of data and materials}

The datasets used and/or analyzed during the current study are available from the corresponding author on reasonable request.

\section{Competing interests}

The authors declare that they have no competing interests.

\section{Funding}

This research did not receive any specific grant from funding agencies in the public, commercial, or notfor-profit sectors.

\section{Authorship contributions}

MO designed the study, managed and analyzed the data, performed statistical analyses, and wrote the manuscript. TH designed the study and revised the manuscript. TO, TI, KO, SK, and SW critically revised the manuscript. YM discussed the interpretations of the data and revised the manuscript. All authors have read and approved the final manuscript to be submitted. 


\section{References}

1. Dhungana A, Khilnani G, Hadda V, Guleria R. Reproducibility of diaphragm thickness measurements by ultrasonography in patients on mechanical ventilation. World J Crit Care Med. 2017;6:185-9.

2. Beck J, Weinberg J, Hamnegard CH, Spahija J, Olofson J, Grimby G, et al. Diaphragmatic function in advanced Duchenne muscular dystrophy. Neuromuscul Disord. 2006;16:161-7.

3. Boon AJ, Sekiguchi H, Harper CJ, Strommen JA, Ghahfarokhi LS, Watson JC, et al. Sensitivity and specificity of diagnostic ultrasound in the diagnosis of phrenic neuropathy. Neurology. 2014;83:1264-70.

4. Sarwal A, Walker FO, Cartwright MS. Neuromuscular ultrasound for evaluation of the diaphragm. Muscle Nerve. 2013;47:319-29.

5. Scheibe N, Sosnowski N, Pinkhasik A, Vonderbank S, Bastian A. Sonographic evaluation of diaphragmatic dysfunction in COPD patients. Int J Chron Obstruct Pulmon Dis. 2015;10:1925-30.

6. Heinzmann-Filho JP, Vasconcellos Vidal PC, Jones MH, Donadio MV. Normal values for respiratory muscle strength in healthy preschoolers and school children. Respir Med. 2012;106:1639-46.

7. Spiesshoefer J, Herkenrath S, Henke C, Langenbruch L, Schneppe M, Randerath W, et al. Evaluation of respiratory muscle strength and diaphragm ultrasound: normative values, theoretical considerations, and practical recommendations. Respiration. 2020;99:369-81.

8. Reiter M, Totzauer A, Werner I, Koessler W, Zwick H, Wanke T. Evaluation of inspiratory muscle function in a healthy Austrian population-practical aspects. Respiration. 2006;73:590-6.

9. Gopalakrishna A, Vaishali K, Prem V, Aaron P. Normative values for maximal respiratory pressures in an Indian Mangalore population: a cross-sectional pilot study. Lung India. 2011;28:247-52.

10. Goligher EC, Fan E, Herridge MS, Murray A, Vorona S, Brace D, et al. Evolution of diaphragm thickness during mechanical ventilation. Impact of inspiratory effort. Am J Respir Crit Care Med. 2015;192:1080-8.

11. Terminology, Diagnostic Criteria Committee, Japan Society of Ultrasonics in Medicine. Standard method for ultrasound evaluation of carotid artery lesions. J Med Ultrason (2001). 2009;36:219-26.

12. Coiffard B, Riegler S, Sklar MC, Dres M, Vorona S, Reid WD, et al. Diaphragm echodensity in mechanically ventilated patients: a description of technique and outcomes. Crit Care. 2021;25:64.

13. De Bruin PF, Ueki J, Bush A, Khan Y, Watson A, Pride NB. Diaphragm thickness and inspiratory strength in patients with Duchenne muscular dystrophy. Thorax. 1997;52:472-5.

14. Szeinberg A, Marcotte JE, Roizin H, Mindorff C, England S, Tabachnik E, et al. Normal values of maximal inspiratory and expiratory pressures with a portable apparatus in children, adolescents, and young adults. Pediatr Pulmonol. 1987;3:255-8.

15. Wilson SH, Cooke NT, Edwards RH, Spiro SG. Predicted normal values for maximal respiratory pressures in caucasian adults and children. Thorax. 1984;39:535-8.

16. Pines A, Kaplinsky N, Olchovsky D, Rozenman J, Frankl O. Pleuro-pulmonary manifestations of systemic lupus erythematosus: clinical features of its subgroups. Prognostic and therapeutic 
implications. Chest. 1985;88:129-35.

17. El-Halaby H, Abdel-Hady H, Alsawah G, Abdelrahman A, El-Tahan H. Sonographic evaluation of diaphragmatic excursion and thickness in healthy infants and children. $\mathrm{J}$ Ultrasound Med. 2016;35:167-75.

18. Boussuges A, Gole Y, Blanc P. Diaphragmatic motion studied by m-mode ultrasonography: methods, reproducibility, and normal values. Chest. 2009;135:391-400.

19. Noda Y, Sekiguchi K, Kohara N, Kanda F, Toda T. Ultrasonographic diaphragm thickness correlates with compound muscle action potential amplitude and forced vital capacity. Muscle Nerve. 2016;53:522-7.

\section{Tables}

Due to technical limitations, Table 1 is only available as a download in the Supplemental Files section.

\section{Figures}

a

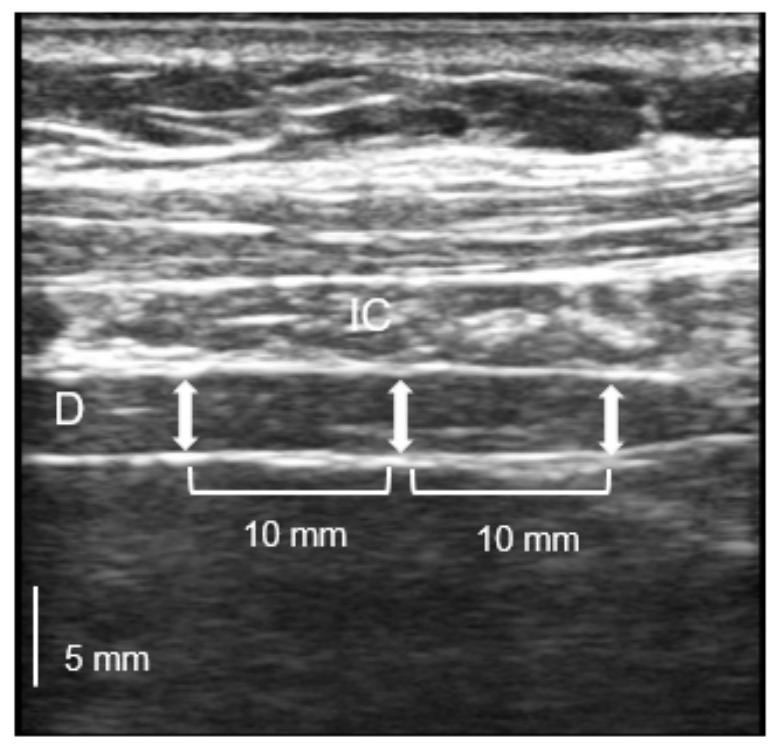

b

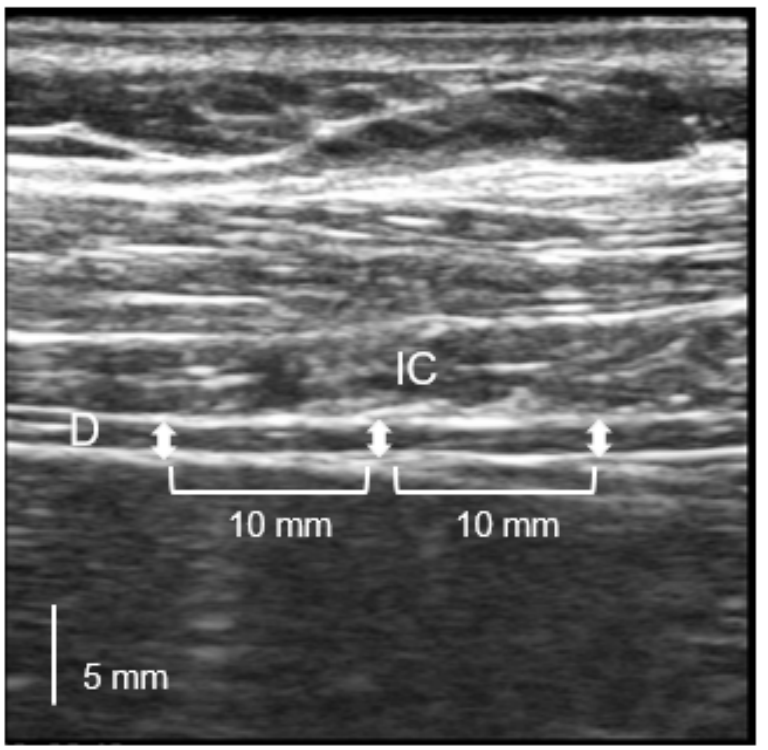

\section{Figure 1}

Representative ultrasound of the diaphragm Diaphragm at total lung capacity (a) and functional residual capacity (b). The diaphragm ( $D$, white bidirectional arrows) is the 3-layered structure situated deep to the intercostal (IC) muscles spanning two ribs; it is identified as the last set of parallel lines, with the pleural and peritoneal membranes overlying the less echogenic muscle. We calculated a mean of three points using the IMT software: the center of the diaphragm thickness, and two surrounding points on either sides (each $10 \mathrm{~mm}$ from the center of the diaphragm thickness). 


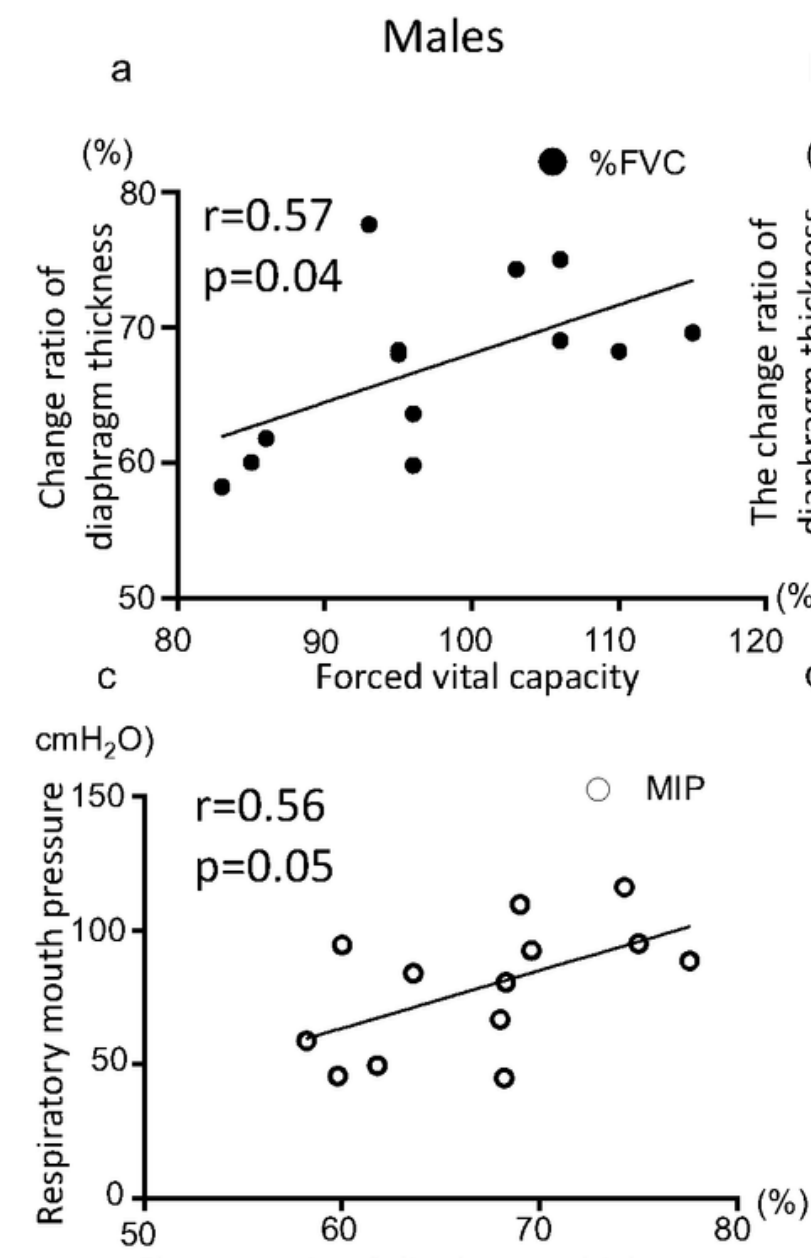

e

Change ratio of diaphragm thickness

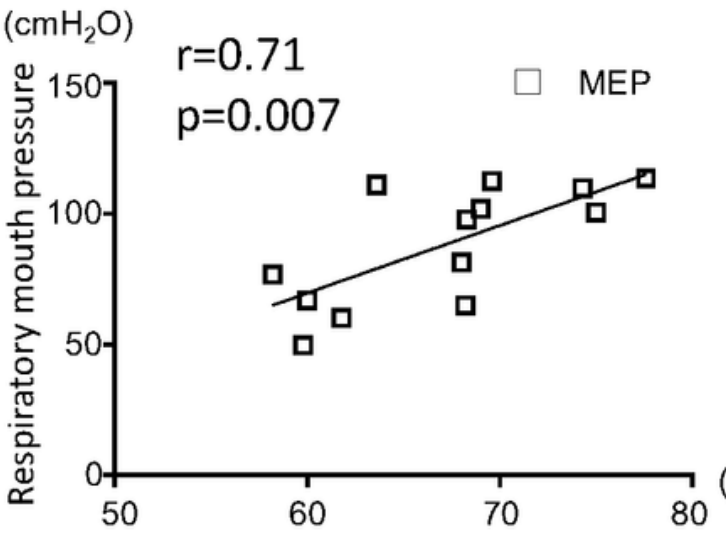

Change ratio of diaphragm thickness b

Females

(\%)

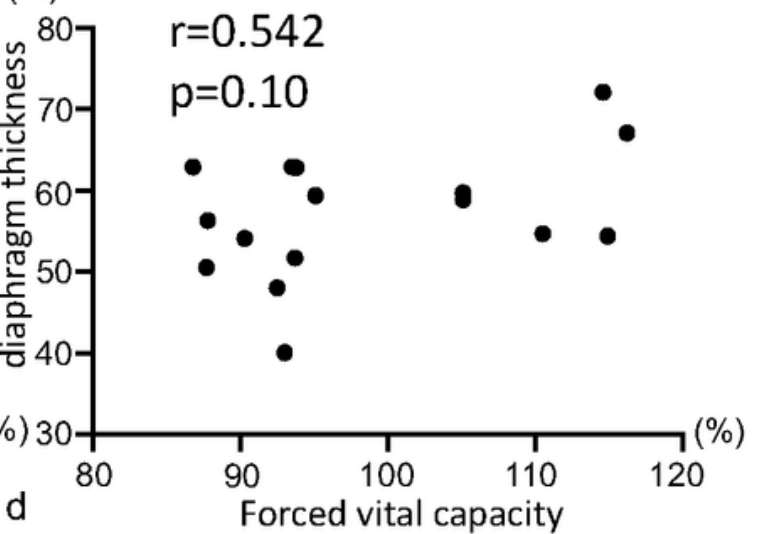

$\left(\mathrm{cmH}_{2} \mathrm{O}\right)$

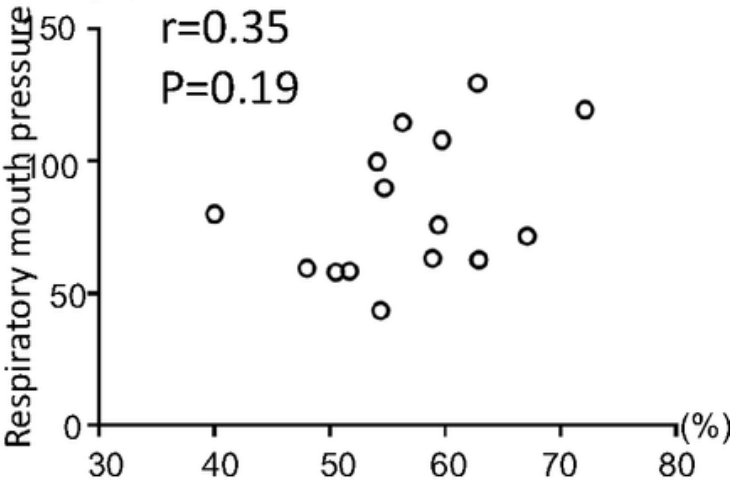

$\mathrm{Ff}$

f

Change ratio of diaphragm thickness

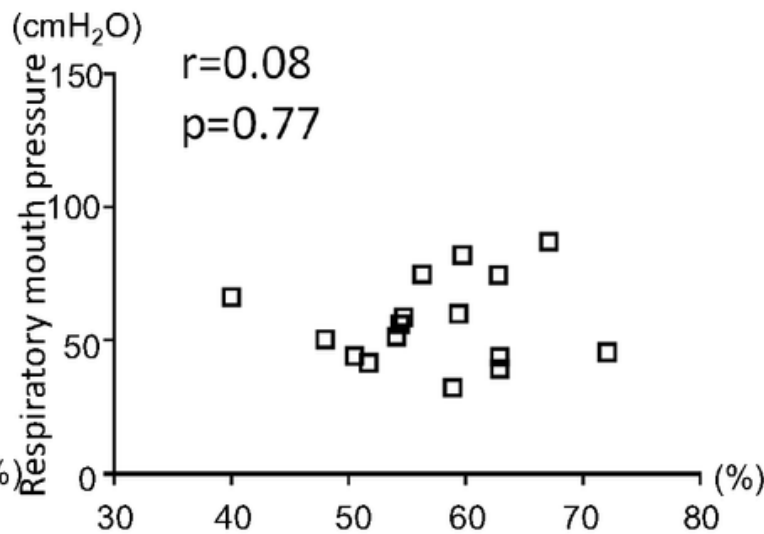

Change ratio of diaphragm thickness

\section{Figure 2}

Correlation between the change ratio of diaphragm thickness and other measures in males and females Measures included \% forced vital capacity, maximum inspiratory pressure (MIP) or maximum expiratory pressure (MEP) for males (a, c, and e), and females (b, $d$, and f). There was a significant positive correlation between the change ratio of diaphragm thickness and \% forced vital capacity or respiratory mouth pressures in males, but not in females. 


\section{Supplementary Files}

This is a list of supplementary files associated with this preprint. Click to download.

- Table1.docx 